\title{
Humane Non-Human Primate Model of Traumatic Spinal Cord Injury Utilizing Electromyography as a Measure of Impairment and Recovery
}

\author{
William A. Graham ${ }^{1,2}$, Douglas L. Rosene ${ }^{2}$, \\ Susan Westmoreland ${ }^{3}$, Andrew Miller ${ }^{3}$, Ervin Sejdic ${ }^{4}$, Shanker Nesathurai ${ }^{1,5,6,7}$ \\ ${ }^{1}$ Division of Physical Medicine and Rehabilitation, Department of Medicine, McMaster University, Hamilton, Canada \\ ${ }^{2}$ Department of Anatomy and Neurobiology, Boston University School of Medicine, Boston, USA \\ ${ }^{3}$ New England Primate Research Center, Harvard Medical School, Boston, USA \\ ${ }^{4}$ Swanson School of Engineering, University of Pittsburgh, Pittsburgh, USA \\ ${ }^{5}$ Department of Physical Medicine and Rehabilitation, Hamilton Heath Sciences, Hamilton, Canada \\ ${ }^{6}$ Department of Physical Medicine and Rehabilitation, St. Josephs Hospital, Hamilton, Canada \\ ${ }^{7}$ Department of Physical Medicine and Rehabilitation, Harvard Medical School, Spaulding Hospital, Boston, USA \\ Email: nesathurai@HHSC.ca
}

Received November 21, 2012; revised December 23, 2012; accepted January 25, 2013

\begin{abstract}
The overall goal of this project is to develop a humane non-human primate model of traumatic spinal cord injury that will facilitate the development and evaluation of therapeutic interventions. The model utilizes neurophysiological techniques to identify the location of the upper motor neuron axons that innervate the lower motor neurons that control tail musculature. This facilitates the placement of a selective lesion that partially disconnects the upper and lower motor neuron supply to the musculature of the tail. An implanted transmitter quantitatively measures electromyography data from the tail. The preliminary data indicates that this model is feasible. The subject was able to tolerate the implantation of the transmitter, without adverse effects. As well, there was no limb impairment, bowel dysfunction or bladder dysfunction. The histopathologic and electromyographic features of the selective experimental lesion were similar to human spinal cord injury.
\end{abstract}

Keywords: Spinal Cord Injury; Animal Model; Pathophysiology; Electromyography

\section{Introduction}

In the USA, there are approximately 250,000 people living with spinal cord injury (SCI). As well, there are approximately 10,000 new traumatic SCI per year [1]. Over the past 50 years, patients are living longer due to improved medical, nursing and rehabilitation services. However, there are currently no pharmacologic treatments that have been demonstrated to improve the ability to walk after traumatic spinal cord injury [2]. One of the challenges in developing and validating potential interventions is the lack of a humane human non human primate (NHP) of SCI.

The overall goal of this project is to develop a humane non-human primate (NHP) model of traumatic spinal cord injury (SCI). This humane NHP model builds on the experimental models that utilized rodents, cats, dogs, and rabbits as well as previous experiments involving monkeys and baboons [3-8]. These previous experiments have yielded valuable information on the histopathology of the initial injury, Wallerian degeneration, spinal cord plasticity, and neural repair. In rodent, dog and cat models, experimental spinal cord lesions have been created by sectioning the spinal cord after surgical exposure of the dura mater, dropping a weight on the spinal cord from a vertical height, compressing the spinal cord with a fixed amount of force, or compressing the spinal cord with a clip.

There are some pathophysiological features of spinal cord injury that are common to human beings, non-human primates, cats, rats dogs and rabbits; however, there are also some substantial differences. For example, cats and dogs with experimental complete transection of the cervical spinal cord, with training, will be able to walk on a treadmill [9-11]. This phenomenon is attributed to spinal stepping, which is mediated by the central pattern generators in the spinal cord. In contrast, human beings and nonhuman primates are unable to walk with complete spinal cord transaction [12,13].

In this context, there is an important role for a humane NHP model of traumatic SCI in evaluating promising new treatments. Levi et al. conclude that the "Cellular 
mechanisms of injury and neural regeneration in primates may substantially differ from those in lower mammals. Differences have been observed in the behavior of human and nonhuman primate cells in neural tissue cultures preparations, as well as in the extensive studies of human SCI” [14]. Tuszynski et al. stated, when comparing rat and primate models, that "the disparate sizes of the spinal cords in the two species, and the potential differences in the extent of the secondary damage cause by such mechanisms as cytokine activation, support a continued need to study primate models" [15]. Tator wrote that experimental spinal cord injury models "should utilize monkeys because of the similarity in the blood supply to the cord in monkeys and humans" [16]. As Courtine et al. have noted in 2007, "The pathway for developing the most effective novel interventions to the greatest number of SCI patients would probably include experiments using nonhuman primates” [17].

To appropriately address the animal welfare issues, this SCI model is predicated on creating small selective neurophysiologically guided spinal cord lesions that partially impair tail movement, but does not impair limb, bladder or bowel function. Based on previously completed motor neuron mapping studies utilizing horseradish peroxidase methods, the optimal location is in the lower lumbar spinal cord. In the Macaca fascicularis, the tail can be considered the monkey's fifth limb; it is used for functional tasks related to mobility, balance and feeding. The tail has as a well-developed musculature and sensory supply, and functions in an analogous manner to a human limb $[18,19]$. In this model, tail movement is measured objectively by a small transmitter that is implanted in the lower back and records electromyographic signals from the tail musculature.

\section{Experimental Methods}

The Harvard University Intuitional Animal Care and Use Committee approved this study. All surgical procedures were completed with sterile technique, under general anesthesia. Vital signs were monitored throughout the entire procedure. Induction will be with ketamine 10 - 15 $\mathrm{mg} / \mathrm{kg}$ intramuscularly. Anesthesia was maintained with inhaled isoflurane at 1 percent MAC. Two surgical procedures were completed. On the first surgical date, a transmitter that measures electromyographic signals was inserted. 30 days later, a second surgical procedure was completed; at this time, a selective spinal cord lesion was created in the lower thoracic spinal cord. Post operative pain was treated with narcotics.

\subsection{First Surgical Procedure}

A midline incision was placed over the lower back, superior to the proximal tail. A small pocket between the subcutaneous fat and underling muscle will be dissected. One small transmitter (D70-EEE, Data Sciences International, St. Paul, MN) that measures electromyographic signals was implanted. A subcutaneous tunnel was created from the back into the tail. A small incision was made on the left and right side of the tail to expose the left and right flexor cauda longus and brevis muscles. An active and reference electrode was sutured on the right and left side of these muscles. The transmitter forwarded electromyographic data via radiofrequency link to a desk top computer. EMG data was processed using MATLAB (Natick, MA, USA). First, the raw EMG signals were filtered using the high-pass Chebyshev Type II filter with the cutoff frequency equal to $10 \mathrm{~Hz}$ in forward and reverse directions to ensure zero-phase digital filtering. The filtering procedure removed artifacts from signals. Next, the signals were segmented signals to detect activation periods using a threshold value equal to three standard deviations of the signal.

\subsection{Second Surgical Procedure}

30 days after the implantation of the transmitter the subject was sedated and given the same anesthesia protocol for this surgical procedure. Disposable surface electrodes were placed on the tail. These electrodes permit the recording of neurophysiological signals. A small incision was completed at the LI level. There, a small laminotomy was performed and a small, insulated radiopaque catheter was advanced, through the laminotomy in the epidural space. The goal is to utilize neurophysiological techniques to identify the precise location of the upper motor neuron axons innervating the lower motor neurons that control tail musculature and facilitate the placement of a selective and limited lesion that disrupts this innervation. To determine the optimal location to induce the lesion, the catheter was positioned where the lowest amount of electrical stimulus results in maximal amplitude of the neurophysiological response at the tail recording site. The goal was to create a lesion that resulted in a 50 percent diminution in the amplitude of the evoked response. Ninety days after the induction of the experimental lesion, the subject was humanely euthanized. The spinal cord was extracted and placed in formalin. The specimens were subject to pathological analysis.

\section{Results}

The first objective of this study was to determine if the subject could tolerate the implanted for a prolonged period of time. In this preliminary experiment, the transmitter was implanted for a period of 120 days. During this time period, the subject tolerated the device, and did not demonstrate any adverse health effects related to the implantation. 
The second objective was to create a selective lesion that did not cause any limb weakness, bowel dysfunction or bladder dysfunction. In this preliminary experiment, the subject did not experience any clinically apparent limb weakness. As well, there was no clinically apparent bowel or bladder dysfunction. There was no clinically appreciable weakness of the tail. However, as anticipated, the selective lesion resulted in electromyographic abnormalities consistent with upper motor neuron injury.

In this model, electromyographic data from the left and right flexor cauda longus and brevis were obtained simultaneously. This is considered an agonist-antagonist pair. The electromyographic data prior to, and subsequent to, the creation of the selective lesion was compared. This analysis demonstrated that the amount of cocontraction of the agonist-antagonist pair increased by approximately 25 percent. This electromyographic finding is consistent with traumatic spinal cord injury. A more comprehensive analysis of the electromyographic features is currently being completed.

The third objective was to create a lesion that had histological features similar to human traumatic spinal cord injury. In this preliminary experiment, the histological features appear similar to human spinal cord injury (please see Figure 1).

\section{Discussion}

The preliminary results in the single subject suggest that it is feasible to develop a humane NHP model of traumatic spinal cord injury. The subject was able to tolerate the implanted transmitter without any adverse effects. As

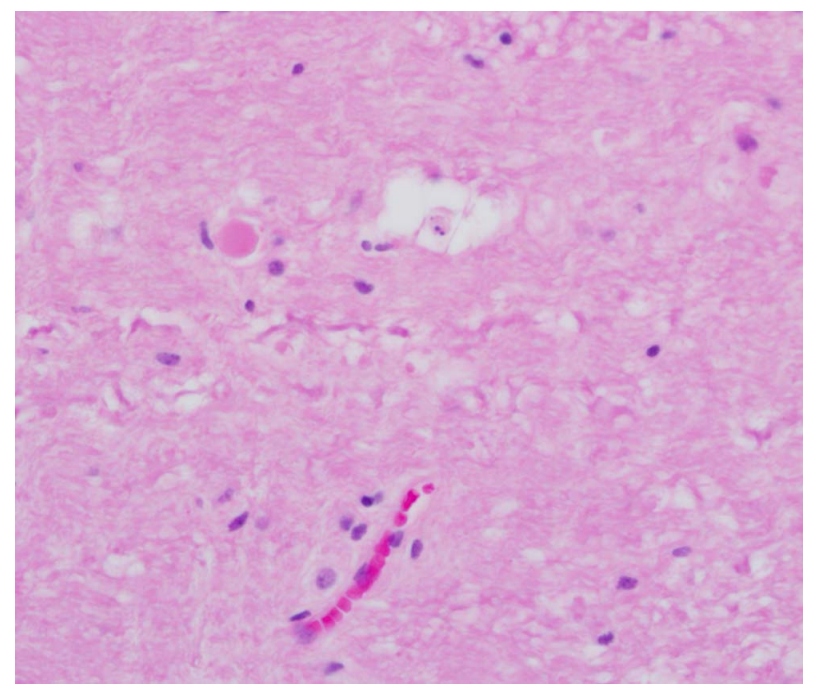

Figure 1. Hematoxylin and eosin stain of spinal cord with light microscopy at $40 \times$ imaging. There is a spheroid, which is associated with a degenerating axon. As well, a digestion chamber is noted, which is consistent with degenerating myelin. These findings are similar to the abnormalities noted in human traumatic spinal cord injury. well, the selective experimental spinal cord lesion did not cause any clinically apparent limb weakness. Furthermore, the selective lesion did not result in any bladder or bowel impairment. The subject was comfortably housed for a period of 120 days. It is important that any proposed model of traumatic spinal cord injury must permit the subject to be humanely housed for an extended period of time. This is particularly important as the benefits of potential pharmacological interventions may become apparent several months after initial treatment.

In this model, the preliminary data suggests that the selective experimental lesions, created by the epidural catheter, have histopathologic features similar to human spinal cord injury [20,21]. Of note, the mechanism for creating the selective spinal cord lesion does not compromise the dura mater, which is consistent with most human spinal cord injuries. As well, a preliminary analysis of the electromyographic data indicates that the lesion results in neurophysiologic impairments consistent with the traumatic spinal cord injury.

The next step is to conduct additional analysis of the electomyographic and histopathologic data. In closing, the preliminary data suggests that it is feasible to develop a humane NHP model of traumatic spinal cord injury.

\section{Acknowledgements}

This work was supported in part by the New England Primate Research Center base grant \# 8P51OD011 103-51.

\section{REFERENCES}

[1] S. Nesathurai, "The Rehabilitation of People with Spinal Cord Injury,” 2nd Edition, AAP Publishing, Whitinsville, 2000.

[2] M. G. Fehlings and D. C. Baptiste, "Current Status of Clinical Trials for Acute Spinal Cord Injury,” Injury, International Journal of Care Injured, Vol. 36, No. 2, 2005, pp. 113-122.

[3] P. Black, R. S. Markowitz, V. Cooper, A. Mechanic, H. Kushner, I. Damjanov, S. D. Finkelstein and K. C. Wachs, "Models of Spinal Cord Injury: Part 1. Static Load Technique,” Neurosurgery, Vol. 19, No. 5, 1986, pp. 752-762. doi:10.1227/00006123-198611000-00006

[4] T. E, Anderson, "Spinal Cord Contusion Injury: Experimental Dissociation of Hemorrhagic Necrosis and Subacute Loss of Axonal Conduction," Journal of Neurosurgery, Vol. 62, No. 1, 1985, pp. 115-119. doi:10.3171/jns.1985.62.1.0115

[5] M. Hashimoto, M. Koda, H. Ino, M. Murakami, M. Yamazaki and H. Moriya, "Upregulation of Osteopontin Expression in Rat Spinal Cord Microglia after Traumatic Injury,” Journal of Neurotrauma, Vol. 20, No. 3, 2003, pp. 287-296. doi:10.1089/089771503321532879

[6] R. Nossin-Manor, R. Duvdevani and Y. Cohen, "q-Space High b Value Diffusion MRI of Hemi-Crush in Rat Spinal Cord: Evidence for Spontaneous Regeneration,” Mag- 
netic Resonance Imaging, Vol. 20, No. 3, 2002, pp. 231241. doi:10.1016/S0730-725X(02)00470-8

[7] G. Bravo, R. Rojas-Martinez, F. Larios, E. Hong, G. Castaneda-Hernandez, G. Rojas and G. Guizar-Sahagun, "Mechanisms Involved in the Cardiovascular Alterations Immediately after Spinal Cord Injury,” Life Sciences, Vol. 68, No. 13, 2001, pp. 1527-1534. doi:10.1016/S0024-3205(01)00952-3

[8] A. Buss and M. E. Schwab, "Sequential Loss of Myelin Proteins during Wallerian Degeneration in the Rat Spinal Cord,” Glia, Vol. 42, No. 4, 2003, pp. 424-432. doi:10.1002/glia.10220

[9] T. G. Brown, "The Intrinsic Factors in the Act of Progression in the Mammal," Proceedings of the Royal Society London, Vol. 84, No. 572, 1911, pp. 308-319. doi:10.1098/rspb.1911.0077

[10] E. Eidelberg, J. L. Story, B. L. Meyer and J. Nystel, "Stepping by Chronic Spinal Cats," Experimental Brain Research, Vol. 40, No. 3, 1980, pp. 241-246. doi:10.1007/BF00237787

[11] J. C. Norreel, J. F. Pflieger, E. Pearlstein, J. SimeoniAlias, F. Clarac and L. Vinay, "Reversible Disorganization of the Locomotor Pattern after Neonatal Spinal Cord Transection in the Rat,” Journal of Neuroscience, Vol. 23, No. 5, 2003, pp. 1924-1932.

[12] E. Eidelberg, J. G. Walden and N. H. Nguyen, "Locomotor Control in Macaque Monkeys,” Brain, Vol. 104, No. 4, 1981, pp. 647-663. doi:10.1093/brain/104.4.647-a

[13] J. F. Fulton and C. S. Sherrington, "State of the Flexor Reflex in Paraplegic Dog and Monkey Respectively,” The Journal of Physiology, Vol. 75, No. 1, 1932, pp. 17-22.

[14] A. D. Levi, H. Dancausse, X. Li, S. Duncan, L. Horkey and M. J. Oliviera, "Peripheral Nerve Grafts Promoting Central Nervous System Regeneration after Spinal Cord Injury in the Primate,” Journal of Neurosurgery, Vol. 96, No. 2, 2002, pp. 197-205.

[15] M. H. Tuszynski, R. Grill, L. L. Jones, H. M. McKay and A. Blesch, "Spontaneous and Augmented Growth of Ax- ons in the Primate Spinal Cord: Effects of Local Injury and Nerve Growth Factor-Secreting Cell Grafts,” Journal of Comparative Neurology, Vol. 449, No. 1, 2002, pp. 88-101.

[16] L. Deecke and C. H. Tator, "Neurophysiological Assessment of Afferent and Efferent Conduction in the Injured Spinal Cord of Monkeys,” Journal of Neurosurgery, Vol. 39, No. 1, 1973, pp. 65-74. doi:10.3171/jns.1973.39.1.0065

[17] G. Courtine, M. B Bunge, J. W. Fawcett, R. G. Grossman, J. H. Kaas, R. Lemon, I. Maier, J. Martin, R. J. Nudo, A. Ramon-Cueto, E. M. Rouiller, L. Schnell, T. Wannier, M. E. Schwab and V. R. Edgerton, "Can Experiments in Nonhuman Primates Expedite the Translation of Treatments for Spinal Cord Injury in Humans?” Nature Medicine, Vol. 13, No. 5, 2007, pp. 561-566. doi:10.1038/nm1595

[18] P. R. Ojha, "Tail Carriage and Dominance in the Rhesus Monkey Macaca mulatta,” Mammalia, Vol. 38, No. 1, 1974, pp. 163-170. doi:10.1515/mamm.1974.38.2.163

[19] W. A. Graham, E. Ludlage, K. Mansfield, D. Magill and S. Nesathurai, "Normative Nerve Conductions in the Tail of Rhesus macaques (Macaca mulatta)," Journal of Medical Primatology, Vol. 35, No. 1, 2006, pp. 25-30. doi:10.1111/j.1600-0684.2005.00136.x

[20] S. Nesathurai, W. A. Graham, K. Mansfield, P. Sehgal, S. V. Westmoreland, S. Prusty, D. L. Rosene and J. B. Sledge, "Model of Traumatic Spinal Cord Injury in Macaca fascicularis: Similarity of Experimental Lesions Created by Epidural Catheter to Human Spinal Cord Injury," Journal of Medical Primatology, Vol. 35, No. 6, 2006, pp. 397-400. doi:10.1111/j.1600-0684.2006.00161.x

[21] A. D. Miller, S .V. Westmoreland, N. R. Evangelous, A. Graham, J. Sledge and S. Nesathurai, "Acute Traumatic Spinal Cord Injury Induces Glial Activation in the Cynomolgus macaque (Macaca fascicularis)," Journal of Medical Primatology, Vol. 41, No. 3, 2012, pp. 202-209. doi:10.1111/j.1600-0684.2012.00542.x 